\title{
A didactic reformulation of the laws of refraction of light
}

\author{
Guadalupe Martínez-Borreguero*1, Ángel Luis Pérez-Rodríguez², María Isabel Suero-López², \\ Francisco L. Naranjo-Correa ${ }^{2}$ \\ ${ }^{1}$ Universidad de Extremadura, Departamento de Didáctica de las Ciencias Experimentales y de las Matemáticas, Avda. de \\ Elvas s/n, Badajoz, España \\ ${ }^{2}$ Universidad de Extremadura, Facultad de Ciencias, Departamento de Física, Avda. de Elvas s/n, Badajoz, España
}

Received on July 23, 2017; Revised on October 08, 2017; Accepted on November 21, 2017.

\begin{abstract}
In this work, an alternative formulation of the laws of refraction of light is presented. The proposed formulation unifies the two established laws of refraction, and it is shown the correspondence between the new and the classic formulations. This new formulation presents a remarkable didactic interest for the conceptual interpretation and resolution of typical problems related to the phenomenon of refraction of light, such as those proposed to students of geometric optics in their first year of college. As an example, this formulation is applied to the resolution of two refraction problems typically assigned to students of such educational level. Results and comments from the students are presented.
\end{abstract}

Keywords: Laws of refraction, new formulation, education, problem-solving.

\section{Introduction}

The refraction of light is a phenomenon widely studied and described traditionally through general notions of waves and rays, and more specifically from an electromagnetic theory point of view. Our students had already studied some concepts about the phenomenon of the propagation of light, as its statement appears in many classical books on general physics or optics [1-7]. In this work, we present a new statement of the law of refraction from an educational point of view, and we show the equivalence to its classic statement. If we consider the traditional widely used books on optics [5], it is mentioned how Newton understood the refraction as the fact that incident light rays "bend or deviate from their path." The traditional statement of the first part of the law of refraction that appears in textbooks, also known as Snell's Law, in honor of Willebrord Snel van Royen (1580-1626), is stated by the following equation:

$$
n_{i} \cdot \sin \theta_{i}=n_{t} \cdot \sin \theta_{t}
$$

where $n_{i}$ and $n_{t}$ are the refractive indexes of the media where the incident and refracted ray are. $\theta_{i}$ and $\theta_{t}$ represent the angles of incidence and refraction, measured from the perpendicular to the plane of separation of the two media at the point of incidence. The second part of the law of refraction states, "The incident rays, the normal to the surface and the refracted rays, lie all in the same plane, called the plane of incidence. In other words, all the wave unit vectors are on the same plane" [5]. Figure 1 shows a typical diagram of the phenomenon

*Correspondence email address: mmarbor@unex.es of refraction of light. This account is similarly stated in other classical books [1-4, 6, 7].

Despite being a set of laws firmly established by its traditional statement on many classical books on general physics and optics, several papers have been published in recent years in various journals with new formulations for the laws of refraction. For example, [8] refers to "the generalized vector laws of refraction". In this paper, the authors point out an ambiguity in the traditional and widely used laws of refraction. Moreover, the authors propose the inclusion of a more refined form of the meaning of the angle of incidence and angle of refraction, incorporating the fundamental definition of angle in geometry.

In a more recent paper [9], the same authors continue the earlier work on the generalized laws of refraction. This work relates the laws of refraction with the principle of conservation of momentum and makes use of Einstein's photon theory.

Later, in [10] the authors apply their new set of generalized laws of refraction to the resolution of two classic problems of optics. Specifically, they analyze what happens to the refracted ray when the refracting surface is rotating, and the solve this problem using the new formulation of the laws of refraction.

In another more recent work, [11], the same authors apply their new set of generalized vector laws of refraction. In their paper, they intend to show the validity of its new formulation using it to solve several cases of image formation using simple refracting surfaces. The aim is to obtain the traditional general lenses formulation from their vector formulation. The case studies carried out 


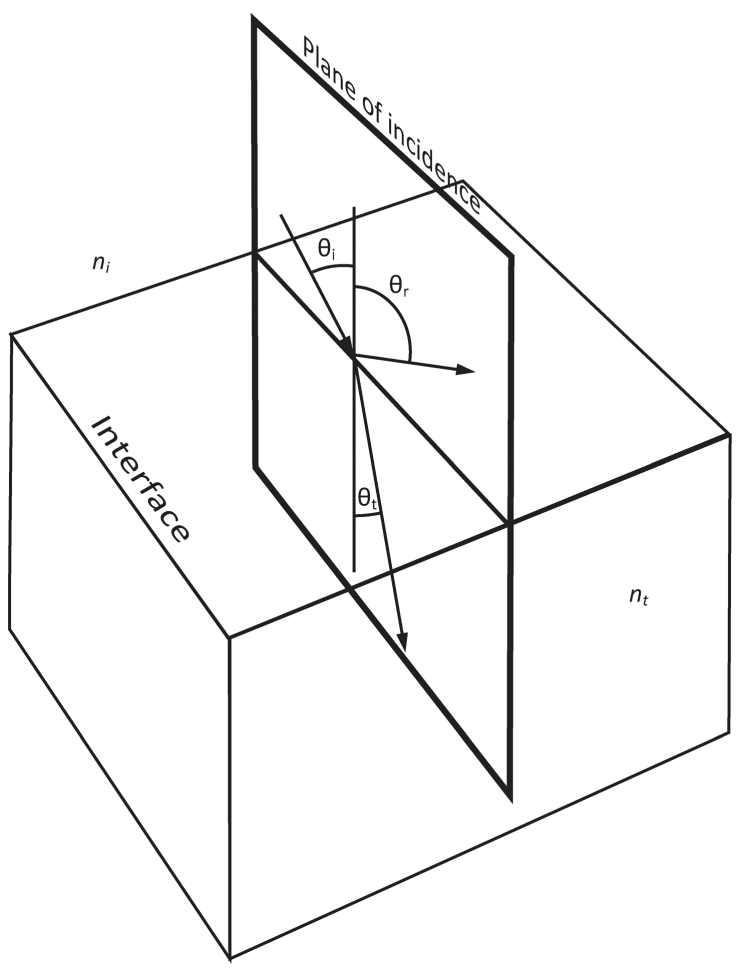

Figure 1: Refraction of light. Light incident at an angle $\theta_{\mathrm{i}}$ at the interface separating two media $n_{i}$ and $n_{t}$ is transmitted into medium $\mathrm{n}_{\mathrm{t}}$ at angle $\theta_{\mathrm{t}}$. The incident and transmitted beams each lie in the plane of incidence [5]

in their paper focus on concave and convex refractive surfaces, with both real and virtual objects.

In another paper [12], other authors have pointed out that, while the laws reformulated by these authors are right, it should be convenient to use the dot product, in addition to the cross product, when expressing the vector laws of refraction. Specifically, they suggest that it is possible to modify the vector laws of refraction stated on [8-11] to incorporate both the cross and the dot product. In this manner wave vectors of the incident and the refracted wave and the normal to the surface are clearly defined, which could improve ray-tracing computations.

Later, paper [13] addresses the issues and questions raised in [12] on generalized vector laws of refraction, establishing a reply to the latter. This study defends that the introduction of the dot product is redundant and confirms the validity of their statements of the generalized vector laws of refraction established in [8-11].

In another paper [14], the authors state that to prove the efficiency of the new generalized laws of refraction they need to arrive at a theoretical proof of Fermat's principle from their statements established on [8]. In this paper, the authors use the case studies of refraction on a flat surface and on a spherical surface, and they successfully deduce the Fermat principle from their own vector laws of refraction.

As a further step forward, the generalized vectorial laws of refraction has been applied in a new paper [15] to produce the generalized vectorial laws of multiple reflection and refraction.

Based on [8] approach, another author [16] has published a formulation of the laws of reflection and refraction. Their new formulas include cases of specular reflection and positive and negative refraction.

In a recent previous work [17] titled "A new formulation of the laws of reflection of light," the equivalence between the new and the classic formulations has been demonstrated. The authors emphasize the significant educational value, as it allows drawing analogies between the phenomena of light reflection and elastic collisions, which is very well known by students.

In line with these recent works, the study presented here proposes a didactic reformulation of the laws of refraction of light, and it shows the equivalence of this new statement with the classic statement. To test the validity of the new formulation, and by way of example, it is applied to solve two traditional optics problems that usually appear on classical optics books.

\section{A didactic reformulation of the laws of refraction of light}

\subsection{Statement}

The statement proposed in this work is as follows:

If at one point on a surface whose orientation in space (or of the tangent plane to the surface, if it is not a plane itself) is defined by a unit vector $\mathbf{k}$ (perpendicular to the surface), strikes and it is refracted an incident ray corresponding to a plane wave (propagating from a homogeneous and isotropic medium into another homogeneous and isotropic medium) whose direction of propagation coincides with that from a unit vector $\mathbf{u}_{\mathrm{i}}$ [expressed in terms of its components with respect to an orthonormal coordinate system, with the z-axis coinciding with the direction of $\mathbf{k}\left(\mathbf{u}_{\mathrm{i}}=\mathrm{u}_{\mathrm{ix}} \mathbf{i}+\mathrm{u}_{\mathrm{iy}} \mathbf{j}+\mathrm{u}_{\mathrm{iz}} \mathbf{k}\right)$, which, along with the y-axis, define the plane of incidence, where $\mathbf{u}_{\mathrm{i}}=$ $0 \mathbf{i}+\mathrm{u}_{\mathrm{iy}} \mathbf{j}+\mathrm{u}_{\mathrm{iz}} \mathbf{k}$ is contained], the refraction occurs such that the refracted ray remains on the plane of incidence, and the component of the unit vector which direction coincides with that of the refracted ray, $\mathbf{u}_{\mathrm{r}}$, parallel to the surface separating the two media, $\mathbf{u}_{\text {ry }}$, equals the product of the relative index of refraction between the two media $\left(\mathrm{n}_{1} / \mathrm{n}_{2}\right)$ and the same component of the incident ray $\left(\mathrm{u}_{\text {iy }}\right)$, i.e., $\mathbf{u}_{\mathrm{ry}}=\left(\mathrm{n}_{1} / \mathrm{n}_{2}\right) \mathbf{u}_{\text {iy }}$." This statement, expressed in everyday language from an educational point of view, is equivalent to saying that "when a ray is refracted, its component parallel to the separation surface between the two media is multiplied by the relative refractive index."

Note: Regarding to the third component, its value is determined by having 1 as the value of the module of the unit vector, also verifying that: $\mathbf{u}_{r}=0 \mathbf{i}+\frac{n_{1}}{n_{2}} u_{i y} \mathbf{j}+$ $\sqrt{1-\left(\frac{n_{1}}{n_{2}} u_{i y}\right)^{2}} \mathbf{k}$

Figure 2 shows a diagram with the notation used to represent the incident and refracted rays, the normal to 


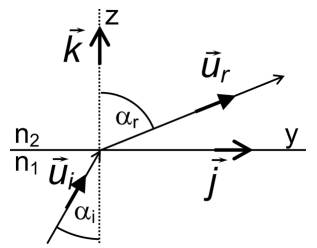

Figure 2: Diagram showing classic refraction

the surface and the angles of incidence $\left(\alpha_{\mathrm{i}}\right)$ and refraction $\left(\alpha_{\mathrm{r}}\right)$.

In short, this is to help students to interpret the meaning of this phenomenon in the sense that when a ray of light propagating in a homogeneous and isotropic medium impinges into another homogeneous and isotropic medium, its unit vector (contained in the plane of incidence) increases one of its components and consequently diminishes another. This modification is determined knowing that the component parallel to the surface separating the two media is multiplied by the relative refractive index between them $\left(\mathrm{n}_{1} / \mathrm{n}_{2}\right)$. That is, $\mathbf{u}_{\mathrm{ry}}=\left(\mathrm{n}_{1} / \mathrm{n}_{2}\right) \mathbf{u}_{\mathrm{iy}}$.

\subsection{Demonstration of the equivalence of the new and classic statements of the laws of refraction.}

In this section, we show the bidirectional equivalence between Snell's law [5] (see Equation 1), and the new statement proposed.

A) If the new statement is right, Snell's law is right as well, and vice versa

Demonstration:

If the new statement is true, and considering that, as seen in Figure 2:

$$
u_{i y}=1 \cdot \sin \alpha_{i}
$$

and

$$
u_{r y}=1 \cdot \sin \alpha_{r}
$$

Then,

$$
\begin{aligned}
u_{r y} & =\frac{n_{1}}{n_{2}} u_{i y} \leftrightarrow \sin \alpha_{r}=\frac{n_{1}}{n_{2}} \sin \alpha_{i} \leftrightarrow n_{1} \cdot \sin \alpha_{i} \\
& =n_{2} \cdot \sin \alpha_{r}
\end{aligned}
$$

as we intended to prove.

Thus, Snell's law is right, and vice versa (as the demonstration can be followed in the opposite direction). That is, the new formulation proposed and Snell's law are equivalent.

This new formulation can be summarized from an educational point of view as "when a ray is refracted, the component parallel to the separating surface of the two media is multiplied by the relative refractive index."

B) If the new proposed statement is right, the second part of the traditional statement of the law of refraction must be correct, and vice versa

If the proposed statement is true, then it is true the part of the traditional formulation of the law of refraction that states "when a light beam is refracted at the interface of two media, the incident beam, the normal to the surface at the point of incidence, and the refracted beam lie all in the same plane, which is called plane of incidence" [18]. Since in the alternative statement we propose the incident beam, the normal to the surface at the point of incidence, and the refracted beam have component $\mathrm{x}=$ 0 , they are coplanar.

If the part of the law of refraction cited in the above paragraph [18] is right, then our new statement is right. That is, if the incident beam, the refracted beam and the normal to the surface at the point of incidence are coplanar, then the plane defined by the incident beam and the normal must be coplanar, which implies that both vectors and the refracted vector have the component $\mathrm{x}=0$, as the new statement proposes.

\subsection{Usefulness of this new statement}

Having demonstrated the equivalence of this new formulation of the laws of refraction and the traditional Snell's law, the new formulation can be used for problem-solving. Consider for example its use in solving problems usually proposed to students at university undertaking a first course on geometric optics.

A) Problem 1: Prove that the beam that emerges after going through a plane-parallel slab has the same direction and sense as the incident beam. Figure 3 shows a diagram of the problem statement.

Solution using the new statement:

$$
\begin{gathered}
\mathbf{u}_{3 \mathrm{x}}=\frac{\mathrm{n}_{2}}{\mathrm{n}_{3}} \mathbf{u}_{2 \mathrm{x}}=\frac{\mathrm{n}_{2}}{\mathrm{n}_{3}} \frac{\mathrm{n}_{1}}{\mathrm{n}_{2}} \mathbf{u}_{1 \mathrm{x}}=\mathbf{u}_{1 \mathrm{x}} \\
\rightarrow \mathbf{u}_{3 \mathrm{y}}=\mathbf{u}_{1 \mathrm{y}} \rightarrow \mathbf{u}_{3}=\mathbf{u}_{1}
\end{gathered}
$$

That is, as we wanted to prove, the emerging beam is parallel to the incident beam.

B) Problem 2: A spotlight is located in the center of the bottom of a three-meters radius cylindrical pool four meters deep, completely filled with water $(\mathrm{n}=$ 1.33). At 30 meters from the edge, there is a hotel. Calculate the minimum height at which a window

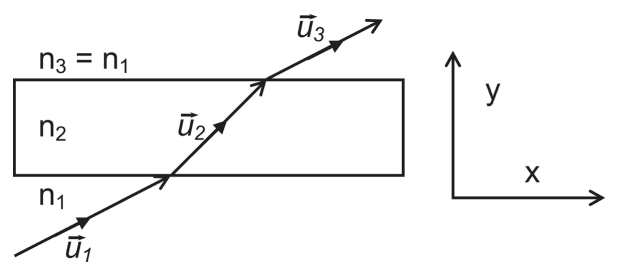

Figure 3: Diagram showing the statement posed in Problem 1 
should be located if we want to see spotlight looking through that window. Figure 4 shows a diagram of the problem statement.

Solution using the new statement:

Using similar triangles: $\frac{\mathbf{u}_{i x}}{1}=\frac{3}{\sqrt{3^{2}+4^{2}}} ; \frac{\mathbf{u}_{r x}}{1}=\frac{30}{\sqrt{30^{2}+h^{2}}} ;$ and, according to our new statement,

$$
\begin{aligned}
\mathbf{u}_{\mathrm{rx}} & =\frac{n_{1}}{n_{2}} \mathbf{u}_{\mathrm{ix}} \rightarrow \frac{30}{\sqrt{30^{2}+h^{2}}}=\frac{1.33}{1} \frac{3}{\sqrt{3^{2}+4^{2}}} \\
& \rightarrow \sqrt{30^{2}+h^{2}}=\frac{30 \sqrt{3^{2}+4^{2}}}{1.33 \cdot 3}=\frac{50}{1.33} \\
& \rightarrow h=\sqrt{\left(\frac{50}{1.33}\right)^{2}-30^{2}}=22.66 \mathrm{~m}
\end{aligned}
$$

Then, the spotlight will only be seen from a window located at 22.66 meters or above.

\subsection{Evaluation of the usefulness of this new statement when solving classic optics problems}

After studying this new statement of the law of refraction and explaining some examples using it, we evaluated if it was useful in solving certain types of optics problems related to light refraction. We used a set of 5 problems of this kind that were proposed to a group of 28 students, and the students could freely use the new statement of the law of refraction or the two traditional laws governing this phenomenon. As a result of this experience, it was found that $78.6 \%$ of students who participated chose to solve the problems using this new statement, which makes clear that it was easier to than the traditional laws. In addition to this objective evidence, we asked the students to make a subjective assessment and to comment on which of the two procedures were they more comfortable with when solving these problems and why. $85.7 \%$ said that to solve this kind of problems they were more comfortable using the new statement. We want to highlight some of the reasons given: "I feel more comfortable operating with segment values than with angle values" and "I find geometry easier than trigonometry."

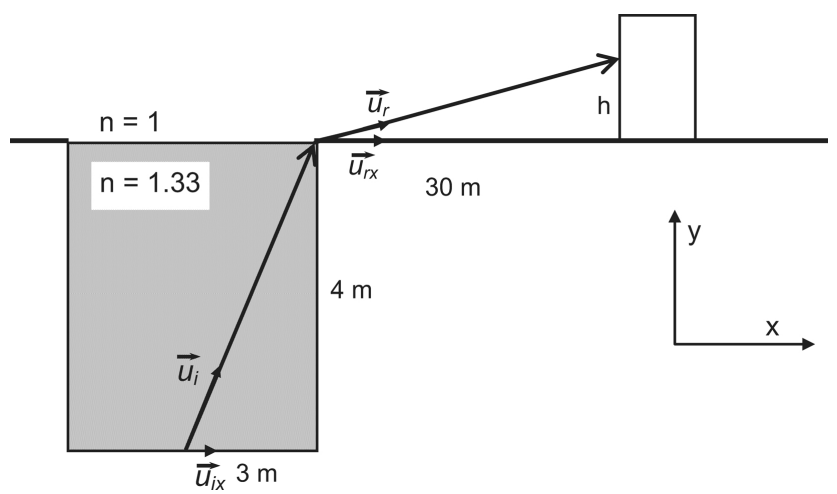

Figure 4: Diagram showing the statement posed in Problem 2

\section{Conclusions}

In this work, we have presented a new formulation of the law of refraction, and it has been proven its equivalence with the traditional statements appearing in classic books of optics.

This new statement can be used to solve classic refraction problems proposed to students at university undertaking a first course on geometric optics.

To prove the validity of this new statement, it is presented, by way of example, the resolution of two classic optics problems. The newly developed statement of the law of refraction allows the resolution of traditional problems of geometrical optics, and in some cases, the solution is much simpler and more intuitive than that obtained using the standard statement of the laws of refraction.

This alternative statement has been of great didactic interest for our students, as it promotes meaningful learning of the refraction of light. The students that learn this statement can solve some problems with more ease.

Both the experimental evaluation and the subjective evaluation performed by the students have shown that most of them believe that the resolution of some problems of this type is easier and more intuitive when using this new statement instead of the traditional statement.

\section{Acknowledgments}

The authors wish to thank the Regional Government of Extremadura for its financial support through Grant GR15102 and GR15009, partially funded by the European Regional Development Fund.

\section{References}

[1] J. Morgan, Introduction to Geometrical and Physical Optics (McGraw-Hill, New York, 1953).

[2] F.W. Sears, M.W. Zemansky, H.D. Young, R.A. Freedman and T.R. Sandin, University Physics (AddisonWesley, Boston, 1999).

[3] F.A. Jenkins and H.E. White, Fundamentals of Optics (McGraw-Hill, New York, 1957).

[4] C. Curry, Geometrical Optics (Arnold, London, 1962).

[5] E. Hecht, Optics (Addison-Wesley, Reading, Massachussetts, 2000).

[6] R.S. Longhurst, Geometrical and Physical Optics (Orient Longman, London, 1974).

[7] P. Drude, The Theory of Optics (Dover, New York, 1954).

[8] P. R. Bhattacharjee, Eur. J. Phys. 26, 901 (2005).

[9] P.R. Bhattacharjee, Optik 120, 642 (2009).

[10] P.R. Bhattacharjee, Optik 121, 2128 (2010).

[11] P.R. Bhattacharjee, Optik 123, 381 (2012).

[12] E.R. Tkaczyk, Optics Letters 37, 972 (2012).

[13] P.R. Bhattacharjee, Optik 124, 2764 (2013).

[14] P.R. Bhattacharjee, Optik 124, 977 (2013).

[15] P.R. Bhattacharjee, Optik 125, 200 (2014).

[16] V.V. Fisanov, Russian Physics Journal 58, 1074 (2015). 
[17] A.L. Pérez-Rodríguez, G. Martinez-Borreguero e M.I. Suero López, Revista Brasileira de Ensino de Fısica 39, e2404 (2017).

[18] S. Burbano de Ercilla, E. Burbano García and C. Gracia Muñoz, General Physics (Tebar, Madrid, 2003). 